\title{
Optimization of Illumination Pulse Duration Increases Flexibility and Performance of Multiphoton Microscopes for Multidisciplinary Research Environments
}

\author{
K. Garsha* and G. Fried*
}

*Imaging Technology Group, Beckman Institute for Advanced Science and Technology, University of Illinois at Urbana-Champaign, 405 N. Mathews Av., Urbana, Il 61801

Multi-photon excitation of electronic states attributed to the simultaneous absorption of two or more photons was predicted in 1931 [1] and first realized in $\mathrm{CaF}_{2}: \mathrm{Eu}^{2+}$ crystals using $0.5 \mathrm{~ms}$ pulses from a ruby laser in 1961 [2]. Thirty years later, the first two-photon excited fluorescence imaging of living specimens was published [3]. Today, near-infrared multi-photon microscopy has become a versatile optical tool with numerous unique advantages owing to the facts that multi-photon absorption is confined to the region of focus, and longer wavelengths of light are used for excitation.

In order to yield the same rate of two-photon excitation, continuous wave excitation requires $10^{2}-10^{3}$ times more average power than pulsed excitation [4]; because of this fact, lasers for multiphoton work typically operate with pulses in the 60 to 200 femtosecond range to achieve an adequate multiphoton excitation effect. In multi-photon microscopy, the fluorochrome excitation rate depends approximately inversely on the input pulse duration [4] [5]. Thus, for a given average power, pulselengthening will decrease the efficiency with which the fluorochrome is excited, and a consequent reduction in the signal to noise ratio will occur (Figure 1). Ultra-short optical pulses have an inherently large frequency bandwidth, and so tend to broaden substantially because of dispersion from propagation through the dispersive elements in a microscope (Table 1). A large part of the induced dispersion is due to the objective, and any relay elements within the microscope will introduce additional dispersion. The reduction of two-photon fluorescence emission as a result of pulse width broadening can be as high as $80 \%$ [6]. The pulse width broadening effect is substantially exacerbated in applications requiring three-photon excitation.

Increasing the average laser power to compensate for pulse lengthening may be undesirable in many instances. Although photodamage in living specimens does not appear to be a result of linear water heating [7] [8], and much photodamage appears to be resultant of the multiphoton excitation process [9] [10], increasing average power to recoup fluorescence intensity does little or nothing to reverse the negative effects of pulse dispersion on important applications such as three-photon excitation [11], and ablation-based nanosurgery [12] [13]. Furthermore, the use of a high average power may also cause problems with optical trapping of cells or organelles [14]. There is very little information available with respect to average power thresholds for induced radiation damage at the biological level for pulse widths below 170 femtoseconds. The present study explores the utility of pulse dispersion precompensation with regard to diverse imaging situations, including biological applications. We use a simple setup consisting of a high index prism sequence [15] [16] [17] (Table 2 ) and a retro-reflector to yield sub-100 femtosecond pulse widths at the objective (Figure 2).

Pulse dispersion precompensation increases the flexibility of a multiphoton system for use in microfabrication studies, including applications that make use of photopolymerization [18] [19] (Figure 3). In these studies, heating due to linear absorption is a major concern, and so the ability to deliver a high peak power in the context of very low average power is paramount. The size of the two and three-photon excitation volume at different numerical apertures is also a primary concern, and the effects of pulse width compensation on the polymerization volume at the focal point for various objectives are explored. 


\section{References}

[1] M. Goppert-Meyer, Ann. Phys. 9 (1931) 273.

[2] W. Kaiser \& C. Garret, Phys. Rev. Lett. 7 (1961) 229.

[3] W.Denk, et al., Science 248 (1990) 73.

[4] C. Xu \& W.W. Webb, J. Opt. Soc. Am. B 13 (3) (1996) 481.

[5] P.E. Hanninen \& S.W. Hell, Bioimaging 2 (1994) 117.

[6] J.B. Guild et al., App. Opt. 36 (1) (1997) 397.

[7]Y.Liu,et al., Biophys. J.68(1995)2137.

[8] A. Schonle \& S.W Hell, Opt. Lett. 23 (5) (1998) 325.

[9] K. Konig et al., Opt. Lett. 24 (2) (1999) 113.

[10] K. Konig et al., Opt. Lett. 22 (2) (1997) 135.

[11] S.W. Hell et al., J. Biomed. Opt. 1(1) (1996) 71.

[12] B.-M. Kim et al., J. Biomed. Opt. 6 (3) (2001) 332.

[13] K. Konig et al., Cell. Mol. Biol. 45 (1999) 195.

[14] A. Schonle \& S.W Hell, Opt. Lett. 23 (5) (1998) 325.

[15] R. L. Fork et al., Opt. Lett.. 9 (5) (1984) 150.

[16] M. Muller et al., J. Microsc. 191 (2) (1998) 141.

[17] C. Soeller and M. B. Cannell, Eur. J. Physiol. 432 (1996) 555.

[18] S. Maruo et al., Opt. Lett. 22 (1997) 132.

[19] H.-B.Sunetal., Appl.Phys.Lett. 79 (10) (2001) 1411.

Table 1. Positive dispersion values (D $\omega)$ per $\mathrm{cm}$ of path length for optical materials @ $\lambda=800 \mathrm{~nm}$

\begin{tabular}{|c|c|}
\hline Material & D $\left.\omega \mathbf{( f s}^{2} / \mathbf{c m}\right)$ \\
\hline Fused Silica & +300 \\
\hline BK-7 & +450 \\
\hline Ti:sapphire & +580 \\
\hline SF-10 & +1590 \\
\hline
\end{tabular}

Table 2. Approximate negative dispersion values $(\mathrm{D} \omega)$ per $\mathrm{cm}$ of path length for Brewster Prism Pairs @ $\lambda=800 \mathrm{~nm}$

\begin{tabular}{|c|c|}
\hline Prism System & $\begin{array}{c}\mathbf{D} \omega \\
\left(\mathbf{f s}^{2} / \mathbf{c m}\right)\end{array}$ \\
\hline $\begin{array}{c}\text { SF-10 Prism Pair } \\
\text { (double pass) }\end{array}$ & -80.2 \\
\hline $\begin{array}{c}\text { BK-7 Prism Pair } \\
\text { (double pass) }\end{array}$ & -12.8 \\
\hline
\end{tabular}

Figure 2. Precompensation for pulsewidth spreading using a pair of dispersive Brewster's prisms.

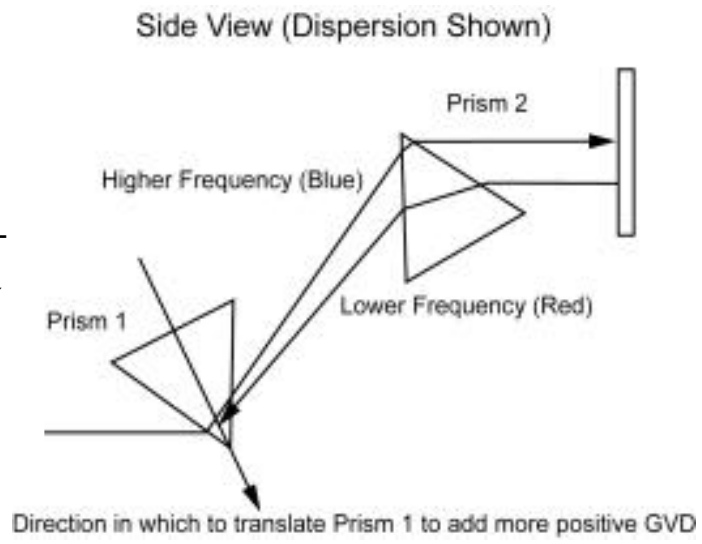

Figure 3. Image of a silica colloid crystal in which the letters UI are written by 3photon polymerization of triacrylate. Below: image shows the $\mathrm{xz}$ axis. Right: image from the xy axis.

ide View (Dispersion Shown)
Figure 1. Relative multi-photon excitation as a function of pulsewidth.

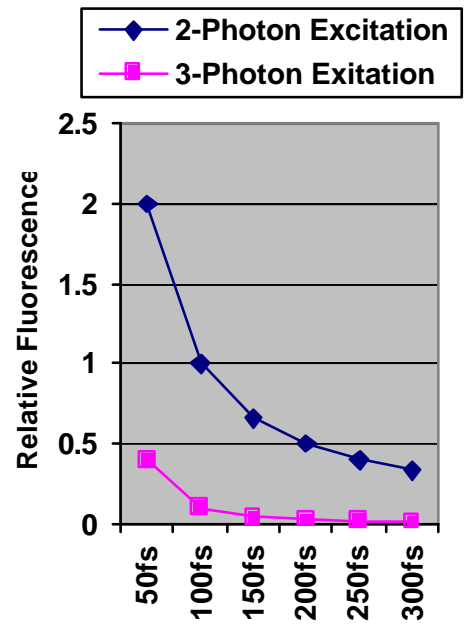

Pulse Width (femtoseconds) 